\title{
Corrigendum: Pulsed Electrical Stimulation of the Human Eye Enhances Retinal Vessel Reaction to Flickering Light
}

\author{
Stefanie Freitag ${ }^{1 \star}$, Alexander Hunold ${ }^{1}$, Matthias Klemm ${ }^{1}$, Sascha Klee ${ }^{1}$, Dietmar Link ${ }^{1}$, \\ Edgar Nagel ${ }^{1,2}$ and Jens Haueisen ${ }^{1}$ \\ ${ }^{1}$ Institute for Biomedical Engineering and Informatics, Technische Universität IImenau, IImenau, Germany, ${ }^{2}$ Ophthalmic \\ Private Practice, Rudolstadt, Germany
}

Keywords: pulsed electrical stimulation, transcranial direct current stimulation (tDCS), flicker light stimulation, dynamic vessel analysis, retinal vessel diameter, vasodilation

\section{A Corrigendum on}

Pulsed Electrical Stimulation of the Human Eye Enhances Retinal Vessel Reaction to Flickering Light

by Freitag, S., Hunold, A., Klemm, M., Klee, S., Link, D., Nagel, E., et al. (2019). Front. Hum. Neurosci. 13:371. doi: 10.3389/fnhum.2019.00371

\section{OPEN ACCESS}

Edited and reviewed by: Felix Scholkmann, University Hospital Zürich, Switzerland

*Correspondence: Stefanie Freitag stefanie.freitag@tu-ilmenau.de

Specialty section: This article was submitted to Brain Imaging and Stimulation, a section of the journal

Frontiers in Human Neuroscience

Received: 14 April 2020 Accepted: 17 April 2020

Published: 13 May 2020

Citation

Freitag S, Hunold A, Klemm M, Klee S, Link D, Nagel E and Haueisen J (2020) Corrigendum: Pulsed Electrical Stimulation of the Human Eye Enhances Retinal Vessel Reaction to Flickering Light. Front. Hum. Neurosci. 14:167. doi: 10.3389/fnhum.2020.00167
In the original article, there was an inaccurate statement. The issue arose in discussions with statistical experts, which encouraged us to publish this correction to avoid confusion to the readers of our article. We made the inaccurate statement that the $t$-test is robust against violation of the normal distribution assumption; however, this only applies under certain conditions. To assure our choice of the paired $t$-test for the data with unconfirmed normal distribution (iTA measurement in the $1200 \mu \mathrm{A}$ group) we used an additional robust method for this data. The results of the robust method that is based on the comparison of the $20 \%$ trimmed mean according to Wilcox (2017) confirm the results of the paired $t$-test.

A correction has been made to the Materials and Methods section, subsection Data Analysis, paragraph 2:

"Measurement results for provocation-induced retinal vasodilation are given as the mean \pm standard error of the mean (SEM) of the groups. Statistical analyses were performed using a statistical software (SPSS Statistics 24, IBM Corporation, Armonk, NY, United States). The ShapiroWilk test was used to check the vasodilation values of each group and each vessel segment for normal distribution. The normal distribution is given for all measurements, except the iTA measurement in the $1200 \mu \mathrm{A}$ group. We performed a paired $t$-test to compare the vasodilation values of the different stimulus conditions. Additionally, we applied a robust method based on the comparison of the $20 \%$ trimmed mean according to Wilcox (2017) for the iTA measurement in the $1200 \mu$ A group because of its unconfirmed normal distribution. All statistical tests were calculated with a significance level of $p=0.05$. Effect sizes were calculated according to Cohen's $d_{z}$ (Cohen, 1988; Lakens, 2013).”

A further correction has been made to the Results section, subsection Retinal Vasodilation, paragraph 2:

"Statistical analyses (Table 1) display differences in the enhancement of vessel dilation after ES+FLS depending on the applied current intensity of electrical stimulation. The $800 \mu \mathrm{A}$ group showed significantly increased vasodilation in all four examined vessel segments after ES+FLS 
compared to FLS. In contrast, the $400 \mu \mathrm{A}$ and the $1200 \mu \mathrm{A}$ group showed an upward trend in mean retinal vasodilation for ES+FLS in all vessel segments but no significant differences. Similar to the paired $t$-test, the additionally applied $20 \%$ trimmed mean comparison for the iTA measurement in the $1200 \mu \mathrm{A}$ group yielded a $p$-value of 0.063 . The effect sizes of the observed effects (Table 1) can be interpreted based on the Cohen classification (Cohen, 1988). Accordingly, the effects of the $800 \mu$ A group were medium to large and the effects of the $400 \mu \mathrm{A}$ and the $1200 \mu \mathrm{A}$ group were small. Additionally, we followed the recommendations given by the CONSORT Group (Moher et al., 2010) and analyzed the differences between stimulus conditions

\section{REFERENCES}

Cohen, J. (1988). Statistical Power Analysis for the Behavioral Sciences, $2 n d$ Edn. Hillsdale, NJ: Lawrence Erlbaum Associates.

Lakens, D. (2013). Calculating and reporting effect sizes to facilitate cumulative science: a practical primer for t-tests and ANOVAs. Front. Psychol. 4:863. doi: 10.3389/fpsyg.2013.00863

Moher, D., Hopewell, S., Schulz, K. F., Montori, V., Gøtzsche, P. C., Devereaux, P. J., et al. (2010). CONSORT 2010 explanation and elaboration: updated guidelines for reporting parallel group randomised trials. BMJ 340:c869. doi: 10.1136/bmj.c869
FLS and ES+FLS. Figure 6 shows these differences including the confidence intervals (confidence level 95\%). Similar to Figure 6, the confidence interval for the $20 \%$ trimmed mean of the iTA measurement in the $1200 \mu \mathrm{A}$ group is [-0.07, 2.21]. Significant differences are indicated by a confidence level that does not include the zero value. This is given for all vessel segments of the $800 \mu \mathrm{A}$ group and is consistent with the results of the paired $t$-test."

The authors apologize for this error and state that this does not change the scientific conclusions of the article in any way. The original article has been updated.

Wilcox, R. R. (2017). Understanding and Applying Basic Statistical Methods Using R. Hoboken, NJ: Wiley.

Copyright (c) 2020 Freitag, Hunold, Klemm, Klee, Link, Nagel and Haueisen. This is an open-access article distributed under the terms of the Creative Commons Attribution License (CC BY). The use, distribution or reproduction in other forums is permitted, provided the original author(s) and the copyright owner(s) are credited and that the original publication in this journal is cited, in accordance with accepted academic practice. No use, distribution or reproduction is permitted which does not comply with these terms. 